\title{
Pharmacovigilance in India, Uganda and South Africa with reference to WHO's minimum requirements
}

\author{
Karen Maigetter ${ }^{1,2 *}$, Allyson M. Pollock ${ }^{3}$, Abhay Kadam ${ }^{4}$, Kim Ward ${ }^{5}$, Mitchell G. Weiss ${ }^{6,2}$
}

\section{Abstract}

Background: Pharmacovigilance (PV) data are crucial for ensuring safety and effectiveness of medicines after drugs have been granted marketing approval. This paper describes the PV systems of India, Uganda and South Africa based on literature and Key Informant (KI) interviews and compares them with the World Health Organization's (WHO's) minimum PV requirements for a Functional National PV System.

Methods: A documentary analysis of academic literature and policy reports was undertaken to assess the medicines regulatory systems and policies in the three countries. A gap analysis from the document review indicated a need for further research in PV. KI interviews covered topics on PV: structure and practices of the system; current regulatory policy; capacity limitations, staffing, funding and training; availability and reporting of data; and awareness and usage of the systems. Twenty interviews were conducted in India, 8 in Uganda and 11 in South Africa with government officials from the ministries of health, national regulatory authorities, pharmaceutical producers, Non-Governmental Organizations (NGOs), members of professional associations and academia. The findings from the literature and KI interviews were compared with WHO's minimum requirements.

Results: All three countries were confronted with similar barriers: lack of sufficient funding, limited number of trained staff, inadequate training programs, unclear roles and poor coordination of activities. Although KI interviews represented viewpoints of the respondents, the findings confirmed the documentary analysis of the literature. Although South Africa has a legal requirement for PV, we found that the three countries uniformly lacked adequate capacity to monitor medicines and evaluate risks according to the minimum standards of the WHO.

Conclusion: A strong PV system is an important part of the overall medicine regulatory system and reflects on the stringency and competence of the regulatory bodies in regulating the market ensuring the safety and effectiveness of medications. National PV systems in the study countries needed strengthening. Greater attention to funding is needed to coordinate and sustain PV activities. Our study highlights a need for developing more systematic approaches to regularly monitoring and evaluating PV policy and practices.

Keywords: Pharmacovigilance (PV), Adverse Drug Reaction (ADR), India, Uganda, South Africa

Copyright: ( 2015 by Kerman University of Medical Sciences

Citation: Maigetter K, Pollock AM, Kadam A, Ward K, Weiss MG. Pharmacovigilance in India, Uganda and South Africa with reference to WHO's minimum requirements. Int J Health Policy Manag 2015; 4: $295-305$. doi: $10.15171 /$ ijhpm.2015.55
Article History:

Received: 7 November 2014 Accepted: 4 March 2015 ePublished: 9 March 2015

*Correspondence to:

Karen Maigetter

Email: karen.maigetter@unibas.ch

\section{Key Messages}

Implications for policy makers

- Given that similar barriers: insufficient funding, limited trained staff, inadequate training programs, unclear roles and poor coordination of activities confronted the Pharmacovigilance (PV) systems of India, Uganda and South Africa, the use of greater capacity-building within the three countries and in other countries with similar levels of healthcare system development would be useful to coordinate and sustain PV activities.

- Having a clear structure of legal PV requirements with corresponding regulations in place where compliance and enforcement could be ensured, would be more effective than reliance on guidelines and normative practice which are not specifically binding.

- The findings contribute to an area that highlights a need for developing more systematic monitoring and evaluation of PV policy and practices on a regular basis (i.e. causality assessment leading to accurate signal detection).

Implications for public

A strong Pharmacovigilance (PV) system is an important part of an overall medicines regulatory system in ensuring safety and effectiveness of medicines. It reflects the stringency and competency of regulatory bodies in the regulation and control of products on the market. The World Health Organization (WHO) has established core minimum requirements for a functional national PV system. Results from a documentary literature search and information obtained from interviews with authoritative officials, professionals and other stakeholders, concluded that only South Africa had a legal requirement for PV and that India, Uganda and South Africa uniformly lacked adequate capacity to monitor medicines and evaluate risks according WHO's standards. Good PV will identify risks associated with medicines in a minimum amount of time and when effectively communicated, will allow for intelligent, evidence-based use of medicines having the potential for preventing many Adverse Drug Reactions (ADRs). The review of national PV systems against the WHO's core minimum requirements has proven an effective way to assess the adequacy of such systems. The World health Assembly should use its law making powers to strengthen and make mandatory PV activities in the interests of public health. 


\section{Background}

The importance of Pharmacovigilance (PV)

Pharmacovigilance (PV) data are vital to ensure ongoing safety and effectiveness of medicines and to provide information concerning regulatory actions such as drug safety alerts, labelling changes to the product information, drug recalls or withdrawal of a drug from the market. PV is defined by World Health Organization (WHO) as: "The science and activities relating to the detection, assessment, understanding and prevention of adverse effects or any other possible drugrelated problems" (1).

Good PV will identify risks associated with medicines in a minimal amount of time and when communicated effectively, information will allow for intelligent, evidence-based use of medicines which will have potential for preventing many adverse reactions. WHO and its regional offices play a key role in supporting countries in promoting the establishment and building of sustainable monitoring systems. It serves as a repository for PV information and disseminates this information appropriately. Under coordination of the WHO and its Collaborating Centre for International Drug Monitoring [the Uppsala Monitoring Centre (UMC) in Sweden], are national centres collecting reports of suspected Adverse Drug Reactions (ADRs) (2). After review, they are sent to UMC for entry into the database which generates signals of previously unrecognized ADRs. The integration of $\mathrm{PV}$ is crucial to the success of public health programs using medicines (3).

National PV and ADR reporting systems in India, Uganda and South Africa are in their infancies and are not yet functioning optimally $(4,5)$. This is due to lack of human, technical and financial resources $(2,6)$. According to the WHO, in many developing countries patients are not adequately safeguarded from accessing harmful and ineffective medicines due to poor PV systems (1). This may result in treatment failures. Particular attention needs to be paid to proper infrastructure and governance, adequate human resources, training and capacity-building and sustainable methodologies and innovation in PV (6). In 2010, the WHO in consultation with its Advisory Committee on the Safety of Medicinal Products (ACSoMP) and the Global Fund to Fight AIDS, Tuberculosis and Malaria (GFATM) agreed on the core the minimum requirements that should be present for a functional national PV system (7).

1. A national PV centre with designated staff (at least one full time), stable basic funding, clear mandates, welldefined structures and roles and collaborating with the WHO Programme for International Drug Monitoring;

2. A national spontaneous reporting system in existence with a national ADR reporting form;

3. A national database or system for collating and managing ADR reports;

4. A national PV advisory committee that is able to provide technical recommendations on safety issues and regulatory actions; validate causality and evaluate risk; and when necessary, participate in crisis management including crisis communication;

5. A communication strategy that is clear for both routine and crises communication.

In 2011, a PV Toolkit was developed and maintained by the WHO Collaborating Centre for Advocacy and Training in PV, University of Ghana Medical School on behalf of the WHO Programme for International Drug Monitoring and in collaboration with the WHO, UMC and ACSoMP. It is a package of simple $\mathrm{PV}$ tools and a description of supporting processes for the conduct of PV (8).

Our study was conceived in the framework of the AMASA (Access to Medicines in Africa and South Asia) project (http:// www.amasa-project.eu/). Similar research had previously been carried out in India and Nepal. To assess the relevance of findings in other low- and middle-income settings of South Asia and Africa, we compared the situation in a large Indian state, Maharashtra, and in two African countries South Africa, which had substantial production capacity, and Uganda which had very limited production capacity. By exploring the regulatory systems and policies in the three countries the effectiveness of PV needed to ensure access to quality medicines could be evaluated. Examining and comparing elements of the three PV systems with reference to the WHO's minimum requirements provides an opportunity to clarify and address limitations of PV and how these affect access and indicate challenges for informed policy-making in these countries and beyond.

\section{Aim}

This study aimed to describe the PV systems in India, Uganda and South Africa. It also aimed to analyse the extent to which the three countries conformed to the minimum PV requirements by the $\mathrm{WHO}$.

\section{Methods \\ Study design \\ Background to study work on Access to Medicines in Africa and South Asia (AMASA)}

A documentary analysis of academic literature and policy reports covering the time period 2005-10 was undertaken to: 1) assess the medicines regulatory systems and policies in India, South Africa and Uganda; and 2) to understand the overall pharmaceutical regulation by looking at regulatory structures and key bodies involved in the process for regulating medicines. The review focused on pharmaceutical regulation, which includes regulatory frameworks and capacity; use of medicines; and PV, including descriptions of the adverse event reporting systems. A gap analysis of the literature indicated a need for further research of the PV systems. An interview guide was designed as the part of the AMASA project for Key Informant (KI) interviews and included questions on regulatory systems and policies with regard to $\mathrm{PV}$ in the three counties.

\section{World Health Organization's (WHO's) Pharmacovigilance (PV) Framework}

The WHO has defined minimum requirements, norms and standards for a functional national PV system (7) and provides a clear authoritative framework for countries developing a PV system (2). This framework was used as a basis for analysing the PV systems in the three countries. $\mathrm{WHO}$ is involved in activities to strengthen country initiatives in PV and safety monitoring. The WHO UMC coordinates the WHO Programme for International Drug Monitoring 
(9). After review of the safety reports, they are entered by the national centres into - VigiBase ${ }^{\mathrm{TM}}$ - a database maintained and developed by UMC on behalf of the WHO. The WHO Uppsala monitoring program recommends that, ideally, a national PV centre should send over 200 reports per million inhabitants per year $(2,10)$.

\section{Literature review process}

Country-specific regulations, policies and guidance for PV were retrieved at the country level using Medline/PubMed, Google Scholar, and ISI Web of Knowledge) for the period 2005-10 by local researchers in India, Uganda and South Africa. Global literature on PV was also retrieved. Search terms included: PV, post-marketing surveillance, drug safety, developing countries, India, Uganda, South Africa and WHO. An additional literature search was conducted following the gap analysis. All the retrieved literature was managed through Reference Manager software.

\section{Key Informant (KI) interviews}

The interviews conducted in the three countries were part of research activities for the AMASA project which included topics covering access to medicines: production, procurement, regulatory practices, $\mathrm{PV}$, counterfeit medicines, drug advertising, interactions with policy-makers and supply chain issues. The questions focusing on PV included: structure and functioning of the program and its challenges, implementation of the system, funding, staffing and training, issues on availability and reporting of data, and awareness and usage of the system. In order to obtain information which was not available in the literature review, the respondents interviewed included high level government officials from the health ministries, national regulatory authorities, members of trade and industry associations, academics, pharmaceutical producers and Non-Governmental Organizations (NGOs) who were knowledgeable and experienced in these areas. The information provided by the respondents represented their viewpoints, based on their experience. It was agreed with the respondents that information they provided would be kept anonymous. The collected information would be coded and respondent names would not be disclosed. Twenty interviews were conducted in India, 8 in Uganda and 11 in South Africa. Ethical clearance was obtained in each country prior to start of the research. The ethical guidelines and procedures were on par with those of the European partners as well as the European Union (EU) directives on the ethical review process. Informed consent in writing was obtained from each respondent and each respondent received written information explaining the research prior to participating in the research project.

\section{Data management, analysis and dissemination}

The interview data was collected using an interview guide. Information from KI interviews was transcribed locally. The interviewer undertook a quality check of the transcriptions. Thematic coding was based on issues identified in the KI interview guide, and these were coded in MAXQDA software by research managers from the three study countries. A lexical search for key words to identify and code additional thematic content from the study was performed.
After reviewing the literature and considering the KI interviews, we formatted a strategy to compare the PV systems of the three countries to the WHO standards. We coded the data sets. The information is presented by country and summarized in Table 1 in the Results. Key findings from the countries were presented at a Dissemination Meeting in London in September 2013. Key opinion leaders from the three countries and $\mathrm{WHO}$ were present who actively participated in a panel discussion.

\section{Results}

The findings for the three countries are separated into: 1) the literature review; and 2) the KI interviews.

\section{India's pharmacovigilance (PV) system \\ Literature review}

In India there was no legal requirement to make PV reporting mandatory. Post-marketing surveillance relied on voluntary reporting. Requirements and guidelines for PV in India were set out in amendment 2005 to Schedule Y of the Drugs and Cosmetic Act (11). It defined the responsibilities of pharmaceutical companies for their marketed products, as well as responsibilities for reporting adverse events from clinical trials and although not explicitly defined, the language was consistent with WHO's definition of PV. A pharmaceutical company holding a product license in India must ensure that it had in place an adequate PV system including establishment and maintenance of appropriate systems to collect collate and evaluate information about suspected adverse reactions. Although not stated in the law, the Periodic Safety Update Report (PSUR) should be submitted by a pharmaceutical company for a product marketed in India. All pharmaceutical companies were required to keep records of ADR reports from marketed drugs and from clinical trials. They were reported to the All India Institute of Medical Sciences (AIIMS) in Delhi. Companies also reported the ADRs to their parent companies via their internal reporting systems.

Attempts had been made since 1986 to introduce a national PV system in India. The latest Pharmacovigilance Programme for India (PvPI) for assuring drug safety was implemented in 2010. The Central Drugs Standard Control Organization (CDSCO), Directorate General of Health Services under the sponsorship of the Ministry of Health and Family Welfare (MHFW), government of India collaborated with the Indian Pharmacopoeia Commission (IPC), Ghaziabad, to coordinate the PvPI $(12,13)$. Despite the new PvPI, PV activities remained under-funded (13).

The IPC which is an autonomous institution under the MHFW also collaborated with the WHO UMC for technical support and to establish the National Coordinating Centre (NCC) AIIMS (12). Targets were set for each of its 5 phases and would continue through 2015 (12). The state drug regulatory authorities were not involved in the PV activities. The Medical Council of India assumed responsibility for the PvPI. It was proposed that every medical college in India should have a PV department of which the vast majority of monitoring centres were in government medical colleges. The three levels of reporting included tertiary centres which reported to the secondary centres which reported to regional 
centres. The regional centres reported to NCC - AIIMS and gave input to the WHO. In India the reporting of ADRs was passive. PV centres were recently established in private sector hospitals (12). Figure 1 indicates the communication pathway of ADRs in India.

The PvPI guidelines stated that physicians, pharmacists and healthcare professionals should report all suspected ADRs on a designated form for submission to CDSCO. India's ADR form was evaluated in a study assessing data capture. Of 18 points which were considered to make a good ADR report, India received thirteen points (14). The International Conference on Harmonization of Technical Requirements for Registration of Pharmaceuticals for Human Use (ICH) Guidelines also requires that ADRs are reported during clinical trials. However, with regard to the general population, poor consumer awareness of the need to report ADRs remained an issue due to a lack of knowledge (15).

India had a formal ADR monitoring system in place since 1986; however a project funded by World Bank revealed that no ADRs had been reported for a period of over 10 years and data which had been collected were never analysed. Much had been attributed to financial inconsistencies (13). Previously, PV had been an externally funded activity. The government had been given a small budget to set up the PV system but this proved impossible for a country the size of India. PV was then positioned in the budget of the Ministry of Health $(\mathrm{MoH})$.

The reporting rate for ADRs was low. India, with its population of approximately 1.24 billion, had a rate of PV reporting below $1 \%(4)$. This low rate was attributed mostly to the lack of training of physicians and pharmacists, and to a poor initiative in reporting ADRs (16). Approximately 45,000 ADRs had been collected, collated and communicated to WHO - UMC which was about 18,000 reports per year from July 14, 2010, when PvPI was launched, through December 2012. The ADR reporting rate for 2011 was approximately 15/ million population $(9,17)$.

\section{Key Informant (KI) interviews}

The head of an ADR monitoring centre stated that resources were not viewed as problematic and that human resources, journals, electronic databases and drug information were available in Mumbai. In contrast, outside of Mumbai there were not enough computers; internet connections were frequently lacking and power outages occurred.

A clinical pharmacologist at an ADR Monitoring Centre stated that in some cases, health managers had no idea about the PvPI and the CDSCO did not keep track of the data due to a lack of human resources. The head of clinical pharmacology at another monitoring centre described that oftentimes the CDSCO had not reviewed the PSUR submitted by the pharmaceutical company so there was a lack of feedback or there was no time to track the post-marketing surveillance. When medical representatives from companies received feedback from the analysed data, it was not known what had been done with the data.

The Head of an ADR monitoring centre described that the previous system depended totally on the poor spontaneous reporting from doctors in Mumbai. However, the new reporting system was also viewed as weak: "Pharmacists are not well-educated about the PvPI and do not or rarely report $A D R s$ or see that they play a role in reporting ADRs".

At one regional centre the situation was different and future physicians were being trained in ADR monitoring. Students were exposed to the ADR reporting form as undergraduates. Physicians and students were making rounds together to monitor patients. Teams were discussing possible ADRs and help was available for physicians. These factors seemed to be motivating the physicians.

Although big hospitals or centres of excellence had PV systems in place, the reporting guidelines were often unclear and cases were often described without further follow-up. An informant explained, as follows: "Patients frequently do not know what medicines they have taken and cannot provide accurate information regarding an ADR. It is not known whether Standard Operating Procedures (SOPs) are in place or how the information are categorized and processed. Doctors often receive information about adverse drug effects from patients and do not have time or are not interested in submitting spontaneous reports. Doctors are afraid that if they report side effects of drugs, they will lose their credibility and will be perceived as prescribing drugs that harm the patient". A local affiliate of a large multinational pharmaceutical viewed the new system as a major initiative and thought $\mathrm{PV}$ would become stronger in India. ADR reports from the Indian population could be analysed and as a result, necessary safety and regulatory decisions could be applied to the

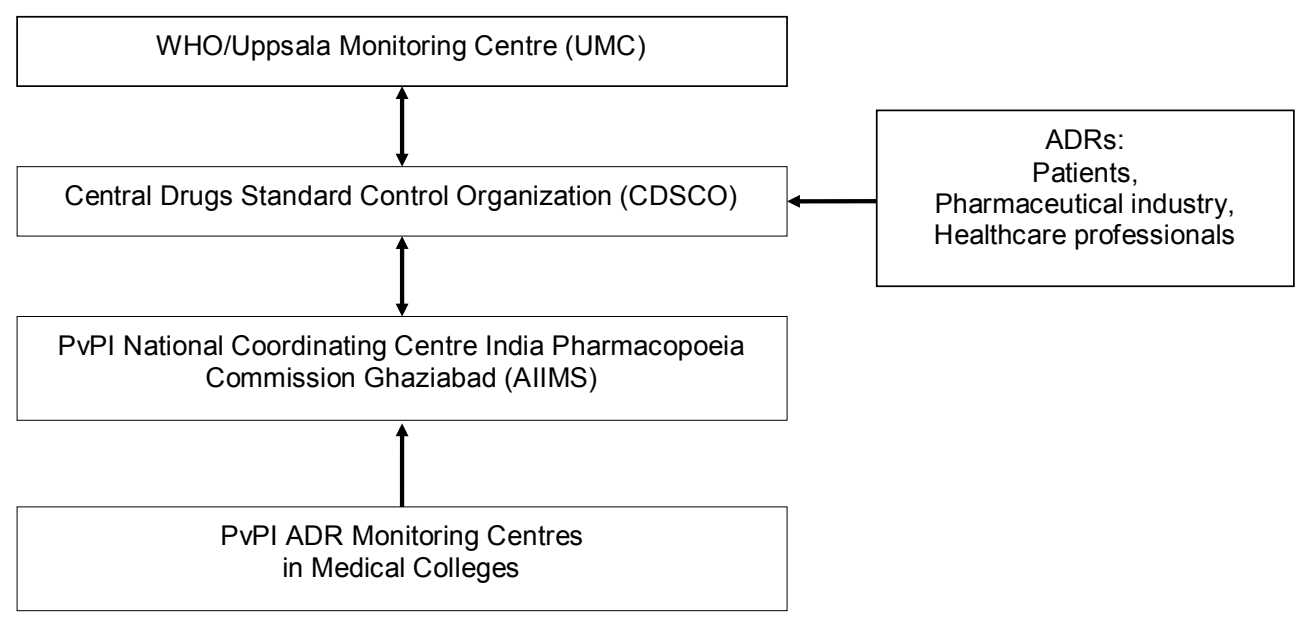

Figure 1. Adverse Drug Reaction (ADR) communication channels in India. 
Indian population.

\section{Uganda's pharmacovigilance (PV) system}

\section{Literature review}

Under the National Drug Policy and Authority (NPD \& A) Act (1993) the Uganda National Pharmacovigilance Centre (NPC) is responsible for PV efforts in the country and mandates the National Drug Authority (NDA) to monitor the safety, efficacy and quality of medicines. The NPD \& A did not specify PV as a law (18). A legal provision mandating $\mathrm{PV}$ and regulations was lacking and a strategy to coordinate stakeholders involved in PV needed developing $(10,18)$. The reporting of ADRs was voluntary for health providers. A draft regulation in 2011 had proposed mandatory reporting of ADRs for industry and healthcare workers (19). It was mandatory for principal investigators of clinical trials to report ADRs based on the "Guidelines for Conducting Clinical Trials" from the National Council of Science and Technology.

The MoH was involved with the PV program at the NDA. The NDA acted as a coordinator and conducted PV surveys. The NPC was one of four core departments reporting to the Executive Secretary/Registrar of NDA and was responsible for managing and co-coordinating PV.

The Drug Information/PV Department of the NDA supervised the NPC activities via facilities at eight regional referral hospitals of university teaching hospitals. Not all regional PV centres were actively engaged in collecting and reporting ADRs. Consistent training was required to encourage centres to increase the reporting rate (10). The Committee on PV and Clinical Trials participated in monitoring effects of medicines (20). A national PV guideline had been developed and distributed to stakeholders (10). Figure 2 illustrates the flow of ADR information in Uganda. A generic form was used for reporting ADRs and it was available in most big hospitals. The NDA, MoH and practitioners cooperated together in PV activities. However, the PV database contained partial sources of information (10). In 2009 the reporting rate was 6 ADRs per million population (19) [approximately 30.6 million population in 2009 (21)].

The NDA had a department supported by technical staff. The
NPC had regular technical support from the WHO country office and shared information with the WHO UMC. Eight staff managed PV issues within the Drug Information/PV Department (19).

The NPC was recently experienced in managing risks and communication. Signal generation and risk evaluation needed to be strengthened. A structured procedure on risk management and risk communication especially for high risk medicines needed to be developed $(18,20)$. Although NPC published newsletters about PV-related activities, press releases, and safety alerts for dissemination of information, effective communication was still a challenge (10).

There was a very limited amount of financial support dedicated for PV and it was calculated to be $<1.0 \%$ of the NDA's budget for 2011-12 (18).

\section{Key Informant (KI) interviews}

A representative of the Pharmaceutical Society of Uganda remarked that it was often difficult to obtain $\mathrm{ADR}$ reports even in public centres due to the low reporting rate; it was a challenge to have the PV system operating smoothly. ADR data were not recorded for many products. The reporting task was perceived as additional work due to all of the forms that needed to be completed.

Money to support PV was being obtained from donors e.g. East African Community, WHO, the United States Food and Drug Administration and from the government. Funding for the PV program was insufficient. The NDA had tried to incorporate PV into educational programs and the concept was currently being included in the curriculum of medical schools.

Outpatient centres were rarely reporting ADRs and reporting forms were not always available. The private sector perceived collecting ADRs as extra work and therefore were reluctant to report events. A local company representative stated: "There is no active PV system in Uganda". It used its own SOP for PV and was conducting its own surveillance.

A senior official of the NDA explained that software provided by WHO was complicated. When the data were not fully entered, the software refused the entry. This discouraged those who wanted to submit reports and the number of reports filed had been declining.

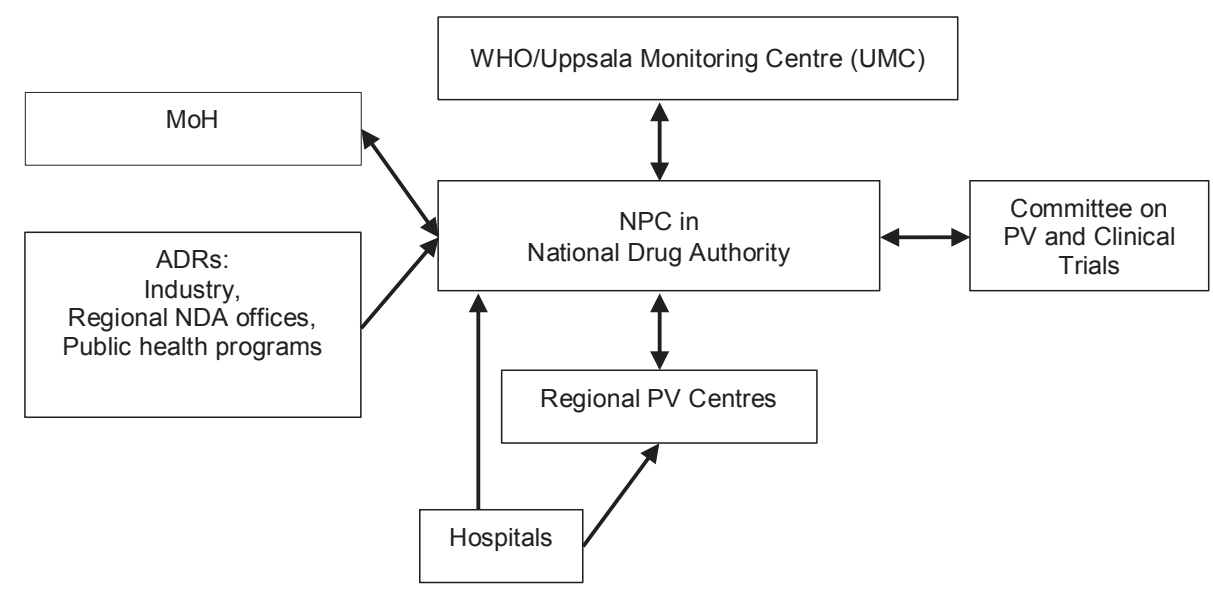

Figure 2. Adverse Drug Reaction (ADR) communication channels in Uganda. Abbrivations: NPC= National Pharmacovigilance Centre; NDA= National Drug Authority; PV= Pharmacovigilance; MoH= Ministry of Health. 
Although staff were being trained through a national training program, there were not enough practitioners to report ADRs. Practitioners may have been interested in PV but there was little incentive for active reporting. The representative of the Pharmaceutical Society remarked that some practitioners had not yet heard of the program.

Oftentimes people had a fear of litigation if they would have been asked to testify as a witness in court cases involving medical malpractice. However, for reporting ADRs a toll-free number had been installed in Kampala and in the rural areas; an SMS system was being developed. On FM radio stations there was information on PV which was being transmitted in the local language to various regions.

\section{South Africa's pharmacovigilance (PV) system Literature review}

$\mathrm{PV}$ is a function of the Medicines Control Council (MCC), the regulatory authority in South Africa. Since 1997, Regulations 34 and 37 of the Medicines and Related Substances Control Act, Act 101 of 1965 as Amended (Act 90) (22) required that the marketing authorization holder report all adverse events, ensure safe use, and collect real-life safety and effectiveness data on a product $(10,22)$.

The MCC had in place the National Adverse Drug Event Monitoring Centre (NADEMC) at the University of Cape Town which was responsible for collating data on the ADR reports and assessment of causality and risk of ADRs. ADRs were reported from the NADEMC to the MCC. Spontaneous ADR reports on antiretroviral drugs were collected solely by the Medicines Information Centre. There were other institutions following up on signals from spontaneous ADRs but they were working independently and information was not being fed into the national system. Other parallel systems existed for public health programs and NGOs but data were not being fed centrally.

The MCC's PV Committee was comprised of a pharmacist and six external experts from various institutions who made up the PV Advisory Committee. It reviewed complaints and ADRs and could make recommendations to the MCC regarding the registration conditions of the specified medicines which included enforcing withdrawal of a medicine from the market and requiring updates to the product information to ensure public safety. It had also drafted guidelines for industry on $\mathrm{ADR}$ reporting. The Clinical Committee became involved if a labelling change was required.

The office of the Registrar, the Medicines Regulatory Affairs (MRA), a Chief Directorate within the Department of Health, provided administrative and technical support to the MCC. The Deputy Director for PV was located within a Directorate of the MRA. Since 2006, in-house regulatory capacity had become an issue in the MRA due to increased workload and many regulatory evaluations were done by PC members.

Pharmaceutical companies worked closely with the NADEMC and ADRs were reported from the NADEMC to the MCC. Spontaneous reports were received from the public, doctors, and pharmacists. The ADR reporting form was also evaluated in a study assessing data capture. Of eighteen possible points considered to make a good ADR report, South Africa's form received a score of twelve (14).

Companies were required to submit PSURs at specified intervals following approval of a drug. The MCC required a company employ a dedicated PV specialist (pharmacist or nurse). Figure 3 shows ADR reporting in South Africa.

The WHO UMC advised that the ADR reporting rate for 2010 was 58/million population (2,902 ADR reports). For 2011 the ADR reporting rate was $77 /$ million population $(4,088$ ADR reports) (23). The population of South Africa in 2010 was approximately 50.1 million (24); in 2011 was approximately 51.8 million (25). Data are often not available or are underreported compared with other countries (5).

\section{Key Informant (KI) interviews}

Several KIs reported that PV activities were fragmented and that duplication existed. The NGOs were not coordinating with the national system and there was under-reporting for Tuberculosis (TB) and anti-retroviral (ARV) drugs. Much of the data that had been collected went directly to the NADEMC where there was insufficient capacity for analysis.

Lack of manpower was identified as the biggest challenge. A chief pharmacist was working for NADEMC with no administrative staff. Two other chief pharmacists, three principal pharmacists and five administrative staff at other locations were working for the National PV Program (NPP) under the coordinator (Deputy Director).

Recruitment of staff for open positions at the NADEMC was

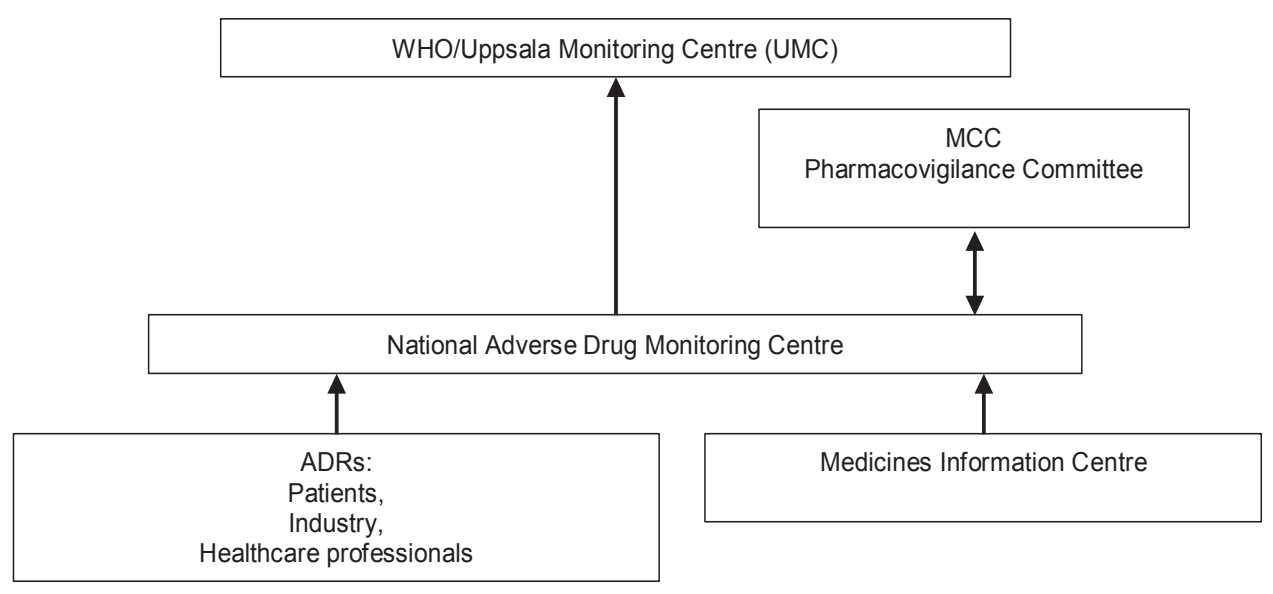

Figure 3. Adverse Drug Reaction (ADR) communication channels in South Africa. MCC= Medicines Control Council. 
very slow and a work backlog of three years existed which was mainly attributed to bureaucratic delays. It was very difficult to find high calibre people in the provinces as PV was perceived as an administrative task. In the future, the NPP was expected to produce many officers, additional training, and a training manual and post graduate courses.

There was no separate budget for PV or meta-analysis and epidemiological studies although it was understood that provision was made for funding requests through MRA. External experts on the PC were remunerated on an hourly basis.

A decentralized PV Plus Program was being planned for the future and clinical interventions from spontaneous reports would be immediately implemented when necessary. Safety information was to be cascaded through other countries and resources would be pooled at a very low cost using the existing infrastructure. Most interventions (e.g. amended labelling and recalls) were a result of safety data from international warnings.

For voluntary reporting healthcare professionals used the standard ADR form available on the MCC website which was viewed by users as cumbersome. Although other forms were accepted, they would often lack sufficient information, often omitting patient details required to track a report.

There was no distinction between the public and private sectors. Reporting was generally low but had increased in the public health sector with more ARV report submissions. Private sector reporting, including private pharmacies was considerably less than in the public sector. Reports from consumers had increased but were not encouraged due to their poor quality and were received mainly from patient support groups or via pharmacies. Poor reporting was also attributed to lack of commitment by health professionals. Private pharmacies made few reports. The NPP identified a need for active surveillance where ADRs could be identified and investigated over a defined period of time Cohort monitoring was done by independent research units and information was not fed into the national system.

Mandatory reporting came from industry as companies were obligated to investigate ADRs. As no guidance existed, reporting was usually done on a case by case basis. One company reported that it did not have capacity to report ADRs electronically and relied on the MCC to manage the data. Faxing ADR reports to the NADEMC was the preferred method of submission. When reports were sent electronically, they created an overload in the system. Data were captured manually and processed slowly. A new Electronic Data Management System (EDMS) was under discussion by MCC and was expected to soon be in effect.

Data were captured as individual reports and a line listing was produced for every PV meeting. Databases used by public health programmes, NGOs and NADEMC were not compatible and data were difficult to access and analyze. Feedback on submitted ADR reports were lacking and the MCC were reluctant to provide information on reporting rates to the provinces, not wanting provinces to make a decision from a signal based on spontaneous reporting. It appears that quantifiable reporting rates were lacking for ADRs. An MCC member stated: "Reports are shared only within the MCC. The Registrar is very reluctant to give any information as it may be interpreted incorrectly. The numbers of reports can be shared with other regulatory authorities but not specific information on individual drugs".

An official from MCC revealed that due to lack of manpower only serious ADRs were reported to the company and the PC did not have the capacity or desire to be involved in the preapproval process or risk analysis.

A regulator from the MCC commented: "International coordination is not functioning well and $P V$ is treated as a local issue. Local ADRs are not often monitored and the Committee depends on data from "E-drug" and occasionally the media. PV is exclusively post-approval. It is mainly spontaneous reporting and from signals received from the USFDA and EMA (European Medicines Agency) although contacts are not as strong as they used to be since the MCC is so slow to respond". The MCC regularly sent out warning letters concerning ADRs for specific drugs but oftentimes information came from other countries.

A provincial department of health official in the pharmaceutical services disclosed that medicine safety issues communicated in the media resulted in an influx of reports on a particular medicine due to the attention they generated. Doctors explained that they did not receive feedback from the MCC concerning reported ADRs while the MCC claimed that there were too many reports on which to provide feedback. Most interventions which resulted in changes in the labelling or product recalls were communicated through a "Dear Healthcare Professional" letter or via a "medical safety alert" in local journals. A drugs or therapeutics bulletin did not exist and the MCC did not have a public relations officer. Drug safety alerts were not sent out. The website of the MCC was not always functional so it was difficult to obtain information.

\section{Summary of Pharmacovigilance (PV) systems}

Table 1 presents a summary of the findings from the literature and KI interviews in the three study countries during the study period. The table compares the conformance of the countries to WHO's standards. The information was cited in: 1) the literature; 2) KI interviews; 3) both; or 4) lacking in both.

\section{Discussion}

Although all three countries had national PV systems in place, none had the capacity to adequately monitor medicines and evaluate risks. Only South Africa had a legal framework in place requiring that adverse events be monitored both actively and passively. India should make its post-marketing surveillance legally mandatory and should not have relied solely on voluntary reporting. Uganda was limited in enforcing its monitoring policies without a legal provision. Placing legal obligations on industry to report to authorities and authorities to pool data within one country or internationally is standard practice in high income countries but few countries place a legal obligation on healthcare professionals to report ADRs. It is questionable as to whether this has demonstrated an increase in reporting. Having a clear structure of legal requirements with corresponding regulations in place would ensure stronger compliance and enforcement rather than relying on guidelines and normative practice which are not specifically binding. 


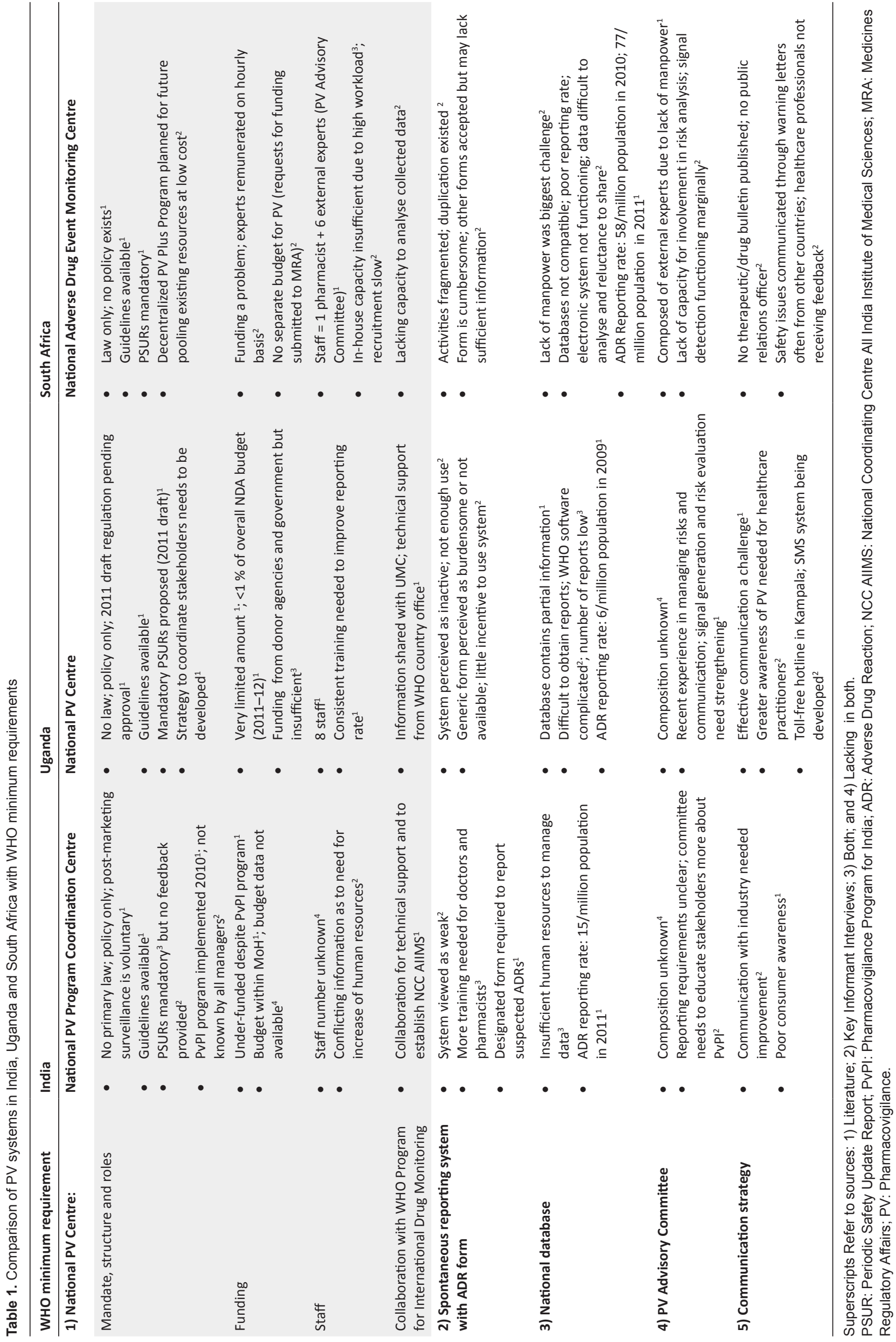


Key deficiencies in all three countries were: lack of sufficient funding and capacity; limited trained staff and training programs; unclear roles; and poor coordination of activities. Although the WHO's country offices and UMC provided collaboration for technical support, more support was needed in order that collected data could be adequately analysed. The WHO minimum requirement of one full time designated staff was insufficient to operate a national PV centre and highlighted a problem with the guidance.

Based on the literature the MCC in South Africa wanted to be seen as being active in PV but the KI interviews revealed that its system was lacking a well-functioning infrastructure to effectively collect and evaluate safety data in a timely manner. Insufficient funding was a recognised problem in all three countries. Budgets were inadequate to meet the needs for effective operation of the systems. Additional funds were required for training of healthcare professionals and for development of educational programs in $\mathrm{PV}$ in the public sector.

The topic PV was not well-integrated into the curricula in medical, pharmacy and nursing schools. However, a regional centre in India was exposing future physicians to the concept of ADR monitoring which was a motivating factor. In Uganda PV was included in the curriculum of medical schools. In South Africa there were plans for additional training in PV and post graduate courses. The countries were in need of continuous support for their efforts if their PV systems were to improve.

Pharmaceutical companies were a major focus of PV activities. Through the submission of PSURS and reporting of serious ADRs within a specified timeframe, companies were required to comply with the regulatory requirements for reporting ADRs to the national PV centres. The local head of a multinational pharmaceutical company in India who viewed India's new system as positive thought that the effects of India's new program would directly benefit the Indian population in the future. To achieve this, it was crucial that all pharmaceutical companies be held accountable for reporting ADR data.

Reporting rates were low in all three countries. This was attributed to lack of capacity, insufficient funding, insufficient training for healthcare professionals and regulators, and for complex software and overloaded systems. Low reporting rates made it difficult for the effective detection of signals, evaluation of risks, and taking necessary regulatory actions. Even though reporting rates were too low, the number of ADR reports was increasing. This indicated that although there was progress, the countries were still struggling with their systems. In all three countries reporting forms did not appear to be standardized, were viewed as cumbersome and therefore contributed little incentive for completion, all of which were problematic.

Advisory committees were adversely affected due to lack of capacity and technical training. It was difficult for them to accurately assess causality. These factors contributed to difficulties in communicating recommendations on safety issues and regulatory actions to healthcare professionals and the public. In Uganda, there had been recent experience in managing risks and communication which demonstrated progress. The decentralized PV Plus Program that was planned for South Africa was expected to improve the flow of safety information. However, the ability of an advisory committee to provide a clear strategy for routine and crisis communication would be negatively impacted without adequate human capacity and technical training.

Without clear communication, a poor awareness of healthcare issues would prevail on the part of healthcare professionals, industry and consumers. Initiatives like the toll-free number and SMS system for reporting ADRs in Uganda and information broadcast on the local radio stations would increase the confidence in the healthcare system.

Other reviews have compared PV systems in Asia (26). Our study has compared these systems in Africa and South Asia but with an explicit focus on the WHO minimum requirements. The deficiencies that our study identified confront low- and middle-income countries which are less of a constraint in developed countries.

Our study relied heavily on KI interviews with authoritative officials, professionals and other stakeholders who had relevant experience in PV. The KIs represented stakeholder interests of health ministries, national medicines regulatory authorities, professional organizations, drug manufacturers, academics and NGOs. Collectively they represented various interests and perspectives with regard to PV, but the depth and quality of their interview responses depended on their interest and willingness to engage thoughtfully in the interview. Recognizing this limitation, we attempted to identify a range of these stakeholders to enable us to assess the consistency and diversity with reference to source of interview data. Nevertheless, details concerning those of budget and staff were not uniformly available. Details regarding the composition and functioning of the national pharmaceutical advisory committees were also limited. Such limitations identified in the course of the study highlight a need for developing more systematic approaches to monitoring and evaluation of PV policy and practices on a regular basis.

The findings from the KI interviews generally confirmed the documentary analysis of the literature although information from the KI interviews often provided greater detail. Major contradictions between the literature and KI interviews were not identified. In the KI interviews one instance was identified where there was conflicting information regarding the need for an increase in human resources.

\section{Conclusion}

The need for a critical analysis presented in this paper was indicated to determine if WHO's minimum requirements regarding $\mathrm{PV}$ were being followed by the three countries during the indicated timeframe. A strong PV system is an important part of the overall medicines regulatory system, indicating the rigor and competence of the regulatory bodies in fulfilling their responsibilities for oversight of producers and markets. Although they had some degree of policy and guidance, India and Uganda did not have a legal framework conforming to WHO's international recommendations. Reporting systems also needed to focus more on active surveillance. Better designed, user-friendly, standardized reporting forms would improve the process of capturing accurate information, including data from spontaneous and 
active reports, and safety reports from clinical trials with reference to $\mathrm{WHO}$ standards.

To achieve these goals, PV needs more particular attention in health science curricula. More training is needed for healthcare professionals and the local pharmaceutical industry. Organizational structures should be strengthened. More funding is required to coordinate and sustain activities. Separate budgets earmarked for PV should be allocated and limitations of human resource capacity in healthcare systems must be overcome.

Inasmuch as the number of ADR reports is increasing, the formulation of WHO standards provides a tool that suggests prospects for improving the effectiveness of PV. Systems for monitoring and evaluation should be implemented to monitor the status and progress in the three study countries with reference to our findings, and implemented elsewhere to enable and promote adherence to basic requirements.

\section{Acknowledgments}

This paper results from research funded by the European Union (EU) Seventh Framework Programme Theme: Health-2009-4.3.2-2 (Grant no. 242262) under the title 'Access to Medicines in Africa and South Asia (AMASA)'. The project team includes partners at the University of Edinburgh (UK), Foundation for Research in Community Health (India), University of Ghent (Belgium), Mbarara University of Science and Technology (Uganda), Makerere University (Uganda), Queen Mary University London (UK), Swiss Tropical and Public Health Institute at the University of Basel (Switzerland) and the University of the Western Cape (South Africa).

The authors wish to thank Paul Waako of Makerere University, Kampala, Uganda and Henry Leng of University of the Western Cape, Bellville, South Africa for their support with the data collection; and to Petra Sevcikova of Queen Mary University of London for her assistance as a scientific adviser for the paper. The authors also wish to thank Christian Schaetti of Swiss Tropical and Public Health Institute for his advice on the technical formatting of the paper.

\section{Ethical issues}

Ethical approval was obtained in each of the countries by the ethical committees of the partner institutions: The Foundation for Research in Community Health, India; Makerere University and Mbarara University of Science and Technology, Uganda; and the University of the Western Cape, South Africa.

\section{Competing interests}

The authors declare that they have no competing interests.

\section{Authors' contributions}

All of the authors played an important role in the acquisition, analysis and interpretation of the data; made substantial suggestions for the revisions of the manuscript; and approved the final submitted version of the paper.

\section{Authors' affiliations}

${ }^{1}$ Department of Epidemiology and Public Health, Swiss Tropical and Public Health Institute, Basel, Switzerland. 'University of Basel, Basel, Switzerland. ${ }^{3}$ Queen Mary University of London, Global Public Health Unit, London, UK. ${ }^{4}$ The Foundation for Research in Community Health, Pune, India. ${ }^{5}$ School of Pharmacy, University of the Western Cape, Bellville, South Africa. ${ }^{6}$ Swiss Tropical and Public Health Institute, Basel, Switzerland.

\section{References}

1. World Health Organization (WHO). The Importance of Pharmacovigilance: Safety Monitoring of Medicinal Products. Geneva: WHO; 2002.

2. Lalvani P, Milstein J. Access to New Health Products in Low Income Countries and the Challenge of Pharmacovigilance. [cited 16 Feb 2013]. Available from: http://pdpaccess.org/ downloads/projects/full-papers/Pharmacovigilance \%20 Discussion\%20Paper.pdf

3. World Health Organization (WHO). The Safety of Medicines in Public Health Programmes: Pharmacovigilance an Essential Tool. Geneva: WHO; 2006.

4. Ghosh AK. Current problems and future aspects of pharmacovigilance in India. Int J Pharm Bio Sci 2011; 2: 15-9.

5. Vaidya SS, Bpharm JJ, Heaton PC, Steinbach M. Overview and comparison of postmarketing drug safety surveillance in selected developing and well-developed countries. Drug Inf J 2010; 44: 519-33. doi: 10.1177/009286151004400501

6. Olsson S, Pal SN, Stergachis A, Couper M. Pharmacovigilance activities in 55 low- and middle- income countries: a questionnaire-based analysis. Drug Saf 2010; 33: 689-703. doi: 10.2165/11536390-000000000-00000

7. World Health Organization (WHO). Minimum Requirements for a Functional Pharmacovigilance System. [cited12 Aug 2013]. Available from: http://www.who.int/medicines/areas/quality_safety/ safety_efficacy/PV_Minimum_Requirements_2010_2.pdf

8. World Health Organization (WHO). Pharmacovigilance Toolkit, version 2.0, 24 January 2012. [cited 25 Apr 2013]. Available from: http://apps.who.int/medicinedocs/en/d/Js19107en/

9. WHO Uppsala Monitoring Centre (UMC). The WHO Programme [internet]. [cited 25 Apr 2013]. Available from: http://www.whoumc.org

10. Strengthening Pharmaceutical Systems (SPS) Program. Safety of Medicines in Sub-Saharan Africa: Assessment of Pharmacovigilance Systems and their Performance. Arlington, VA: Management Sciences for Health; 2011.

11. Central Drugs Standard Control Organization (CDSCO). Schedule $Y$ (amended version) Drugs and Cosmetic Rules. [cited 8 Aug 2013]. Available from: http://cdsco.nic.in/html/ schedule-y\%20\%28amended\%20version-2005\%29\%20original. htm

12. Central Drugs Standard Control Organization (CDSCO). Pharmacovigilance Programme of India (PvPI) for Assuring Drug Safety. Government of India; 2013.

13. Gupta YK. Pharmacovigilance Programme for India [internet]. 2010. [cited 8 Aug 2013]. Available from: http://pharmabiz.com/ NewsDetails.aspx aid $=57406 \&$ sid $=9$

14. Bandekar MS, Anwikar SR, Kshirsagar NA. Quality check of spontaneous adverse drug reaction reporting forms of different countries. Pharmacoepidemiol Drug Saf 2010; 19: 1181-5. doi: 10.1002/pds.2004

15. Adithan C, Dkhar SA. Drug Alert Regional Pharmacovigilance Center. Drug Alert 2012; 6: 2-3.

16. Subhashini DK. Proactive pharmacovigilance vital for drug safety [internet]. [cited 7 Aug 2013]. Available from: http:// saffron.pharmabiz.com/article/detnews.asp?articleid=53054\&se ctionid $=50$

17. Central Drugs Standard Control Organization (CDSCO). Four Years' Achievements 2009-2013. [cited 30 Aug 2013]. Available from: http://cdsco.nic.in/writereaddata/Four\%20years $\% 20$ achievements\%20of\%20CDSCO\%20(2009-2013).pdf

18. Ndagije HB. What is Current Practice of Pharmacovigilance in Uganda? [cited 18 Apr 2013]. Available from: http://africapv2012. files.wordpress.com/2012/04/day-2_5_country-presentationsnras_h-ngadije-uganda-compatibility-mode.pdf 
19. Ndagije HB. Working together with the Public Health Programmes: a regulator's perspective for addressing the minimum requirements for Pharmacovigilance. [cited 13 Aug 2013]. Available from: http://www.nda.or.ug

20. National Drug Authority Uganda. Structure of NDA/Departments/ Drug Information. [cited 26 Apr 2013]. Available from: http://www. nda.or.ug/

21. United Nations Statistics Division. UNdata Uganda. [cited 20 Aug 2013]. Available from: http://data.un.org/Search.aspx?q=uganda

22. Medicines Control Council SA. Medicines and Related Substances Control Act 101 of 1965 after Amendment by the Medicines and Related Substances Control Amendment Act (Act 90 of 1097) Regulations 34 and 37; 1997.
23. Uppsala Monitoring Center (UMC) VigiBase. Numbers of Reports per year in South Africa between 2002 and 2012. Uppsala: Uppsala Monitoring Center; 2013.

24. United Nations Statistics Division. UN data South Africa. [cited 20 Aug 2013]. Available from: http://data.un.org/Search. aspx?q=south+africa

25. South Africa Government. Census 2011 Statistical release P0301.4 /Statistics South Africa. [cited 15 Aug 2013]. Available from: http://www.statssa.gov.za

26. Systems for Improved Access to Pharmaceutical Services (SIAPS). Pharmacovigilance Systems in Five Asian Countries. Arlington, VA: Management Sciences for Health: 2013. 